\title{
A Virtual Holographic Display Case for Museum Installations
}

\author{
Manuela Chessa*, Matteo Garibotti*, Valerio Rossi ${ }^{\dagger}$, Antonio Novellino ${ }^{\dagger}$ and Fabio Solari* \\ *Department of Informatics, Bioengineering, Robotics and System Engineering - DIBRIS, University of Genoa, \\ Via all'Opera Pia 13, 16145 Genova, ITALY \\ Emails: \{manuela.chessa,matteo.garibotti,fabio.solari\}@unige.it \\ ${ }^{\dagger}$ ETT SpA, Via Sestri 37, 16154 Genova, ITALY \\ Emails: \{valerio.rossi,antonio.novellino\}@ettsolutions.com
}

\begin{abstract}
Today, it is important in society to make artworks accessible to mass audiences and to widen participation in culture. In such a context, virtual reality is one of the areas of greatest interest: new devices and new techniques are affordable for many users, and virtual and real worlds are often mixed together. In this paper, we propose a "virtual holographic" display, i.e. a stereoscopic virtual reality system that is able to replicate the behavior of a real showcase for exhibitions. It works in a completely virtual manner and it can yield to a new generation of entertainment "holographic" installations. We evaluate such a system through an experimental session with 20 users. In particular, we compare the proposed system, based on a stereoscopic technique (TD3D), with respect to a standard motion parallax technique in terms of the users' perceptual experience.
\end{abstract}

Keywords-Virtual Reality, stereoscopic visualization, motion parallax, entertainment and education, cultural heritage

\section{INTRODUCTION}

To make culture accessible to the general public is an important aspect of the modern society, and, in particular, to make available the artworks can be a way to allow the diffusion of the traditions and the cultural heritage of a nation. This can be possible if we are able to remove the physical constraints that confine them in specific locations, and to promote better access to and wider participation in culture for enhancing cultural diversity and intercultural dialogue [1].

Recent years have seen a very rapid increase of interest in technologies and systems related to the field of Virtual Reality (VR). The entertainment applications have supported the development of VR systems, and now application areas, which were not interested in the past, have started to look to VR with greater interest, to keep up with the times and offer more innovative proposals. This interest led to the development of new tools, both software and hardware, to deliver contents and experiences more and more fascinating and reliable. Graphics engines are able to handle very complex scenes, with a rendered visualization and a management of physics at a realistic level. New hardware devices allow users to enjoy content in a more immersive way, and to interact with virtual objects in a natural way (e.g. Microsoft Kinect ${ }^{1}$ ). In particular, in the application domain of cultural heritage, the VR can be used as a tool for restoration of artworks [2], or for preserving historical places that may be destroyed [3]. In [4], [5] how VR technologies have been used in museums, to

\footnotetext{
${ }^{1}$ Kinect: http://www.microsoft.com/en-us/kinectforwindows/
}

create virtual copies of artworks and exhibitions so that they may be appreciable even by remote users or at a later time, is presented. Moreover, the VR applications can be used for education and public understanding of science [6], [7], [8].

Among the available technologies for visualization, several authors focused on the development of 3D displays without the need of wearing special glasses: in particular, the issue of creating holographic displays is addressed by following several approaches. In [9], an interactive multi-user holographic display, which allows freely moving observers to share a 3D scene, is presented. The approach is based on a specially arranged array of projectors and a holographic screen. In [10], the authors presented a full-color 3D display system, based on high density directional images. A new kind of installations for museum that produces 3D hologram in a small volume is exemplified by the DREAMOC ${ }^{2}$ products series. Using transparent and reflective glasses, this kind of products is able to project images onto real object, providing a sense of coexistence between real and virtual objects that mimics holographic images. Other examples of devices useful for museums are the Heliodisplay ${ }^{3}$ and the Displair Fog ${ }^{4}$ that projects images on a flow of steam about the size of a monitor. Thanks to this technique, the images appear to be floating in mid-air, making the effect of a hologram, but this particular technique does not allow a good image quality.

In this paper, we present a "virtual holographic" system that is intended to simulate the structure of a glass case inside which there is an object that can be observed from different points of view by an observer moving around it. In particular, our aim is to propose a low-cost and scalable system, characterized by high performances in terms of balance between both costs and visual perception, with respect to the previously cited systems. It is worth noting that our technique differs from the previously described approaches, since it is based on standard 3D monitors (or 3D TV), thus no special hardware is necessary for its implementation. The correct perception of virtual objects (i.e. without distortions and misperception issues [11]) is obtained through the TD3D stereoscopic technique [12], [13] that, by tracking the user positions, is able to propose to him/her virtual situations, which replicate the perception of real objects within a display case.

\footnotetext{
${ }^{2}$ RealFiction: http://www.realfiction.com

${ }^{3}$ IO2 Technology: http://www.io2technology.com/

${ }^{4}$ Displair: http://displair.com
} 


\section{The Virtual Holographic System}

We developed our system by considering the following aspects. (i) Scalability: the proposed system must be easily reproducible and adaptable with respect to the intended use and the available budget. The display devices may vary from $3 \mathrm{D}$ active monitors, bigger passive $3 \mathrm{D}-\mathrm{TV}$, up to $3 \mathrm{D}$ projection systems. Even the devices responsible of the user's tracking can range from low-cost systems such as the Microsoft Kinect, up to more complex and expensive motion capture systems. (ii) Customization: the proposed system should be suitable for different setups. The number of monitors, the size of the area in which virtual objects will be placed, the possibility of interacting with the objects, and the space in which the users can move must be easily settable.

In the following, we describe two techniques to produce the 3D perception of virtual objects, the calibration and registration of the real and virtual reference frames, and the hardware and software components of the system.

\section{A. The TD3D stereoscopic technique}

It is important to focus on the aspects that allow the proposed system to best replicate the behavior of real objects in a virtual environment, mimicking the holographic effect. The technique we have used has been proposed in [12], [13]. Such a technique enables of the $3 \mathrm{D}$ visualization by means of the creation of correct stereoscopic images, and it allows a moving observer to perceive 3D virtual objects as similar as possible as they would be in reality, in terms of location, size and dynamical behavior. Figure 1 shows how different stereoscopic projections are created with respect to the different positions of the eyes of a moving observer. It is worth noting that this technique needs an accurate detection of the eyes' position in order to correctly work.

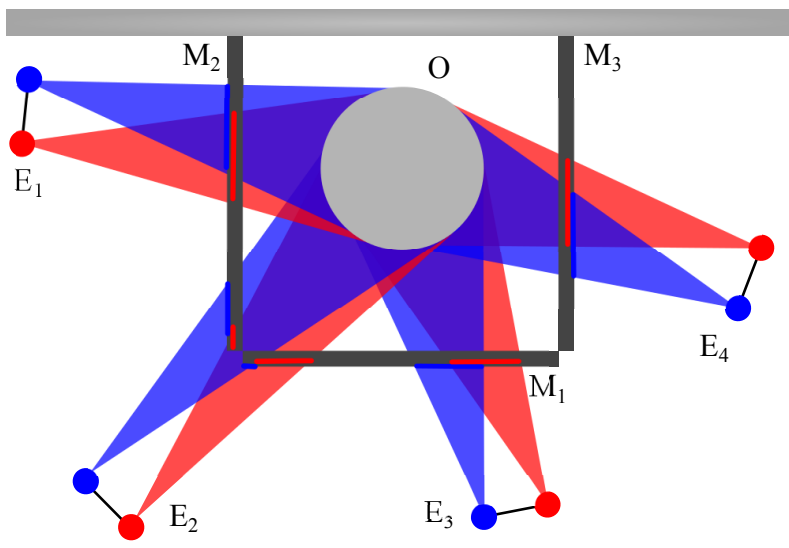

Fig. 1. Sketch of four stereoscopic projections depending on the user's position: $M_{1,2,3}$ are three stereoscopic monitors, $E_{1,2,3,4}$ are four different possible positions of the user's eyes, $O$ is the virtual object being displayed, and the red and blue triangles represent the stereoscopic projections of the same virtual object for each user's position.

To obtain stereoscopic projections that allow a user to perceive a realistic virtual scene, we have to detect the position of the user's eye and to place the virtual stereo cameras in the correct positions. To implement such a behavior, we use generalized asymmetric frustums, since the standard asymmetric frustums, used in the off-axis technique, are not able to produce the correct rendered images for a moving observer. Figure 2 shows the generalized asymmetric frustums $\left(F_{-} M_{1} E_{R}\right.$ and $F \_M_{1} E_{L}$ for the $M_{1}$ monitor, and $F_{-} M_{2} E_{R}$ and $F_{-} M_{2} E_{L}$ for the $M_{2}$ monitor) for two monitors and the stereoscopic projections (only two points are highlighted) of a virtual object that is seen on both monitors (cf. position $E_{2}$ of Fig. 1)

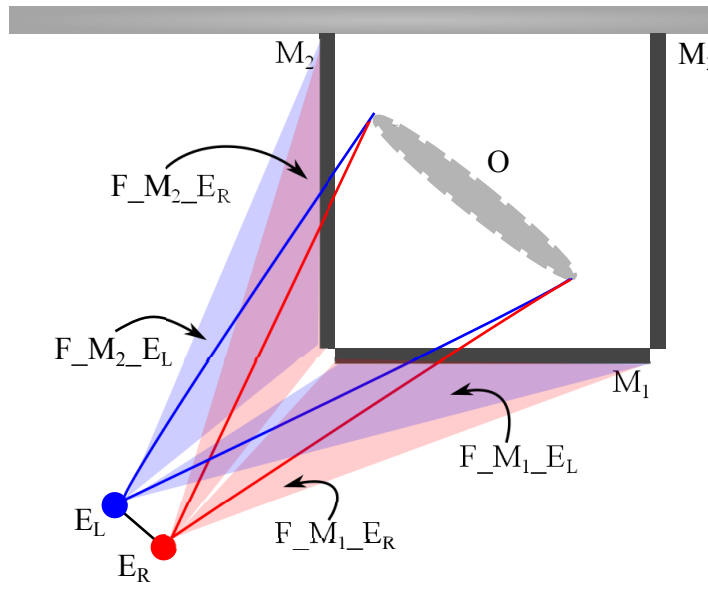

Fig. 2. Sketch of generalized asymmetric frustums. The position $E_{L}$ and $E_{R}$ of the user's eyes, which are in the same location of the virtual stereo cameras, are shown. The stereoscopic projections of the virtual object $O$ are highlighted by the red and blue solid lines. The left and right generalized asymmetric frustums for the two monitors $\left(F_{-} M_{1} E_{R}\right.$ and $F_{-} M_{1} E_{L}$ for the $M_{1}$ monitor, and $F_{-} M_{2} E_{R}$ and $F_{-} M_{2} E_{L}$ for the $M_{2}$ monitor) are computed and generated at the same time.

In particular, the monitor surface and the focal plane of the related generalized asymmetric frustum must be always coincident, thus the focal plane is defined by three 3D corners of the monitor. Moreover, the position of the virtual stereo cameras (i.e. the vertices of the frustums) must be in the same location of the user's eyes. It is worth noting that a rototranslation of the standard off-axis frustums (e.g., [14]) does not correctly address the problem, since the focal plane and the monitor must coincide even when the two eyes are not parallel to the monitor. In the proposed system multiple pairs of generalized asymmetric frustums have to be computed and generated simultaneously, since the user sees multiple monitors at the same time.

\section{B. The motion parallax technique}

By following a procedure similar to the one described in the previous section, we can also implement a system that exploits the motion parallax (instead of stereoscopic visualization) to induce the perception of 3D to the users [15], [16]. Again, there is the need of generalized asymmetric frustums (though they are not stereoscopic) in order to correctly project images onto the monitors from the user's point of view.

\section{The calibration procedure}

We developed a procedure that allows an easy calibration and registration of the devices to properly set up the proposed virtual reality system. Several chessboards are used to calculate 
the intrinsic and extrinsic parameters of the devices. An external calibrated camera captures snapshots of the chessboards, and then we compute the transformations that relate their reference frames. The calibration procedure, shown in Figure 3 , is an extension of the one proposed in [17]. For the sake of clarity, we describe the calibration procedure by taking into account only two monitors.

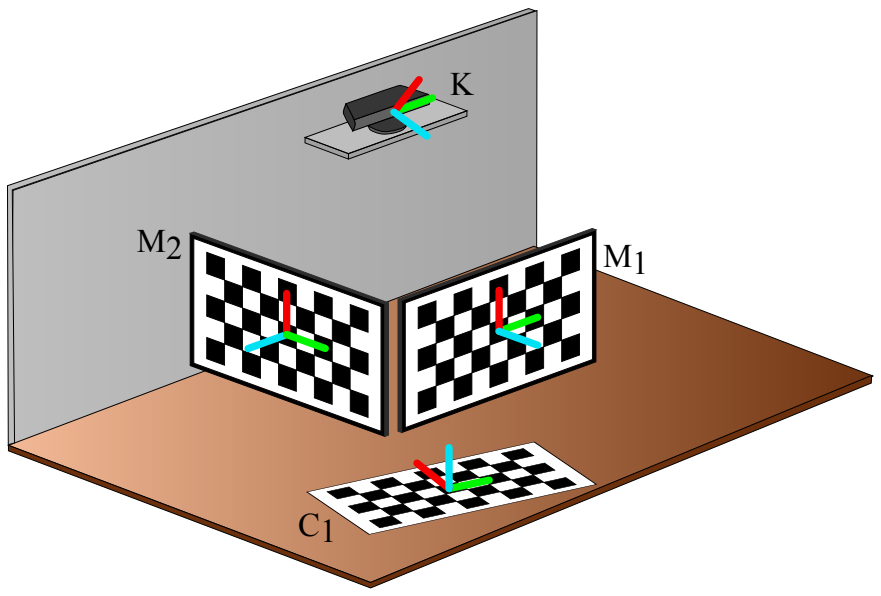

Fig. 3. The calibration rig. $K$ is the tracking device, $M_{1}$ and $M_{2}$ are two $3 \mathrm{D}$ monitors, on which virtual chessboards are rendered, and $C_{1}$ is a real chessboard.

In order to compute the correct generalized asymmetric frustums for each monitor, we need to know the 3D eyes' positions, and the 3D monitor sizes and positions in the same reference frame.

In Figure 3, $M_{1}$ represents the primary monitor, corresponding with the center of the reference system with respect to which it is necessary to know the positions of the other devices. Moreover, we consider the reference frame $W$ of the $M_{1}$ monitor coincident with the one of the virtual world, since the focal plane of the generalized asymmetric frustum is on the monitor surface.

In order to know the position of the monitor $M_{2}$ with respect to reference system $W$, two full-screen chessboards of known size are displayed on both the monitors; in this way the center of the chessboard corresponds precisely to the center of the monitor. The two chessboards are then captured by a calibrated camera, and the roto-translation $\left[T_{M_{1} M_{2}} ; R_{M_{1} M_{2}}\right]$ between the two monitors' reference frames is computed accordingly, giving the relation

$$
\mathbf{x}_{W}=R_{M_{1} M_{2}} \mathbf{x}_{M_{2}}+T_{M_{1} M_{2}}
$$

where $\mathbf{x}_{M_{2}}$ denotes a point in the $M_{2}$ monitor's reference frame, and $\mathbf{x}_{W}$ represents the same point in the virtual world's reference frame, which is coincident with the reference frame of the $M_{1}$ monitor.

The same procedure can not be applied to the tracking device (Microsoft Kinect). The on-board camera of the tracking device itself is used to compute the roto-translation between a real chessboard and the device. However, it can not directly see the virtual chessboard placed onto the $M_{1}$ monitor, since in our setup the considered tracking device is placed above the monitors. To solve this, we use an additional chessboard $C_{1}$ : first we compute the roto-translation $\left[T_{M_{1} C_{1}} ; R_{M_{1} C_{1}}\right]$ between the $M_{1}$ monitor and the additional chessboard $C_{1}$, then the roto-translation $\left[T_{C_{1} K} ; R_{C_{1} K}\right]$ between the chessboard $C_{1}$ and the tracking device $K$, giving the consecutive roto-translation

$$
\begin{gathered}
\mathbf{x}_{C_{1}}=R_{C_{1} K} \mathbf{x}_{K}+T_{C_{1} K} \\
\mathbf{x}_{W}=R_{M_{1} C_{1}} \mathbf{x}_{C_{1}}+T_{M_{1} C_{1}}
\end{gathered}
$$

where $\mathbf{x}_{K}$ denotes a point in the reference frame of the Kinect, $\mathbf{x}_{C_{1}}$ is its representation with respect to the reference frame of the chessboard $C_{1}$, and $\mathbf{x}_{W}$ is its representation with respect to the virtual world reference frame.

\section{The hardware components}

Our aim is to obtain a system easily scalable and based on off-the-shelf devices that allow us to obtain a low-cost system (e.g. ten times cheaper than the Heliodisplay device). The system is based on:

- Three BENQ XL2720Z, 27 inch stereoscopic 3D monitor.

- $\quad$ Kinect 2 for Windows as tracking device.

- Windows PC with i7 processors, 8GB RAM, Nvidia Quadro K4200.

- Nvidia Vision Pro glasses.

As future implementations, we can consider the use of Leap Motion $^{5}$ as a device for handling the users' interaction with the system, and the use of $3 \mathrm{D}$ projectors to eliminate the troublesome presence of the edges of the monitors.

\section{E. The software components}

The software developed to implement the proposed system is composed of two parts: one for the visualization of the stereoscopic VR environment, and the other for the tracking of the user's eyes location.

As graphics engine we use Unity Game Engine ${ }^{6}$, since it provides high performance, outstanding graphics, and it can be customized, e.g. by implementing the TD3D technique that is necessary for the correct stereoscopic rendering of virtual objects to a moving observer (see SectionII-A). In addition, we have increased the functionality of Unity by creating some scripts to interface the different parts of the virtual reality system, such as the reading of the positions provided by the tracking devices, and to easily manage the integration of information on the number and size of monitors and their positions. Figure 4 shows the output of the Graphics Engine for the proposed system, from the point of view of an user located in front of the edge of the "virtual holographic" case (position $E_{2}$ represented in Fig. 1).

With regard to the tracking of user's eye position, we decided to use the Microsoft Kinect 2 as a capture device, and we took advantage of its Skeleton Tracking and Face Tracking capabilities: this allows us to have a quite robust tracking of eyes' positions with an acceptable framerate of $30 \mathrm{fps}$.

\footnotetext{
${ }^{5}$ Leap Motion: https://www.leapmotion.com/

${ }^{6}$ Unity 3D: http://unity3d.com/
} 


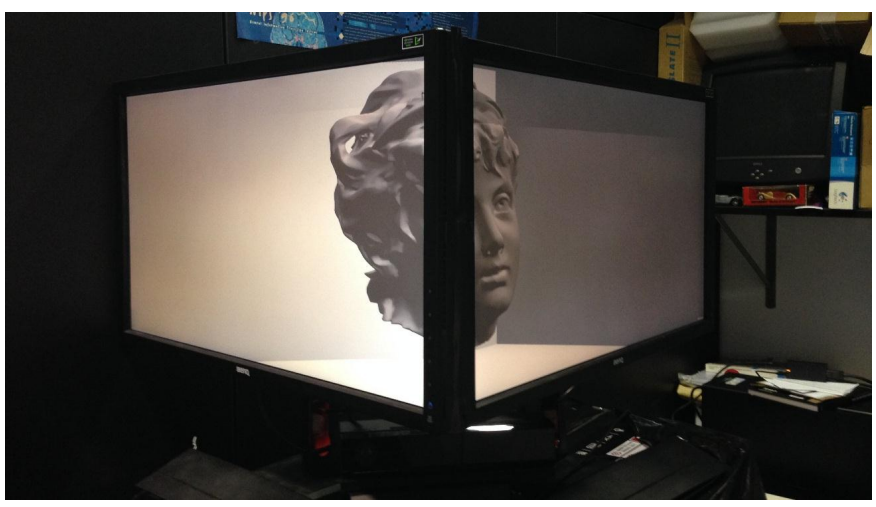

Fig. 4. Snapshot of the proposed "virtual holographic" case, on which the stereoscopic projections of a virtual artwork are displayed from the point of view of an user located in a position similar to $E_{2}$ of Fig. 1.

All the communications among the different components of the proposed system are achieved by using the VRPN protocol [18]. This allows us to run the different modules of the system on different PCs, thus reducing the need of having very high performance workstations. Moreover, it is easy to substitute single modules of the system.

\section{EXPERIMENTAL EVALUATION}

To validate the quality of the proposed "virtual holographic" system, we created an experiment, which was attended by 20 subjects: they had observed a virtual statue displayed by our system, as if it were in a glass case in a museum. Ten subjects saw the virtual statue through the described stereoscopic technique with continuously updated generalized asymmetric frustums (TD3D technique), other ten subjects through a standard parallax technique. The participants were both male and female, with ages ranging from 20 to 40 , and with normal or corrected-to-normal vision. They were not aware of the actual technique used to display to them the virtual contents. At the end of each session, they were asked to answer a questionnaire with 9 closed-ended questions. Subjects rated their feelings on a 5-point Likert scale, where 1 indicated negative feeling at all and 5 indicated the most positive experience. The questions listed below were related to eye tiredness (Q1-Q2), qualitative perception of the virtual objects (Q3-Q6), and technical issues (Q7-Q9).

Q1 How clear is your vision?

Q2 Ho do your eyes feel?

Q3 How realistic is the scene?

Q4 Are you able to observe the object from different points of view?

Q5 How different is it with respect to a real museum display case?

Q6 Do you perceive distortions?

Q7 Do you perceive latencies?

Q8 Is the object stable?

Q9 Do you enjoy the session?
The distribution and the statistics of the responses to the nine questions, for the two techniques, are reported in Figure 5 .

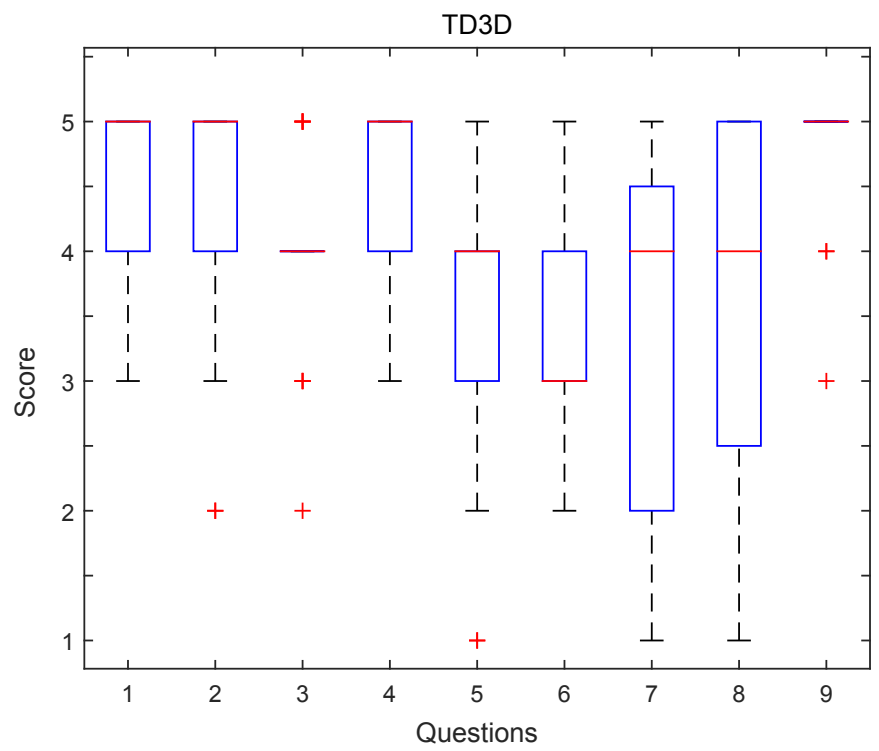

(a)

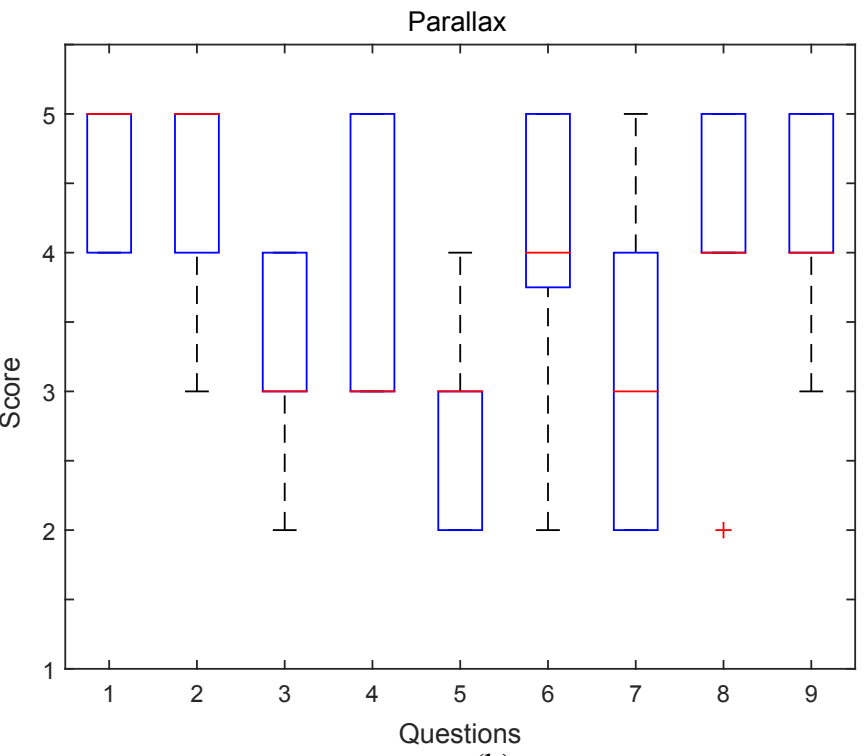

(b)

Fig. 5. Box plots of the scores given by the subjects to the 9 questions, for the "virtual holographic" display implemented through the TD3D stereoscopic technique (a), and a standard motion parallax technique (b). The red lines represent the median scores, the blue boxes are scores between the first and the third quartiles.

The goals of this experiment are: (i) to assess the performances of the proposed "virtual holographic" display, implemented trough the TD3D technique, with respect to a standard parallax technique, in terms of correct perception of the virtual objects; (ii) to verify the effects of the proposed technique with respect to visual fatigue. To examine these, we averaged across subjects the scores for the TD3D and the parallax techniques (see Fig. 6). The dark and light red bars represent data for the two techniques. The reported scores were on average higher (thus indicating a better feeling) with the TD3D technique for 
Q1, Q3, Q4, Q5, Q7, and Q9. The differences were statistically significant (Wilcoxon signed-rank test, $p<0.025$, one-tailed) for Q3 (realism of the scene), Q4 (possibility of observing the object from different points of view), Q5 (realism with respect to a real museum case), and Q9 (general appreciation of the experience). There were no significant differences for questions Q1 and Q2 (eye tiredness). It is worth noting that the stereoscopic TD3D technique is more affected by stability issues $(\mathrm{Q} 8)$.

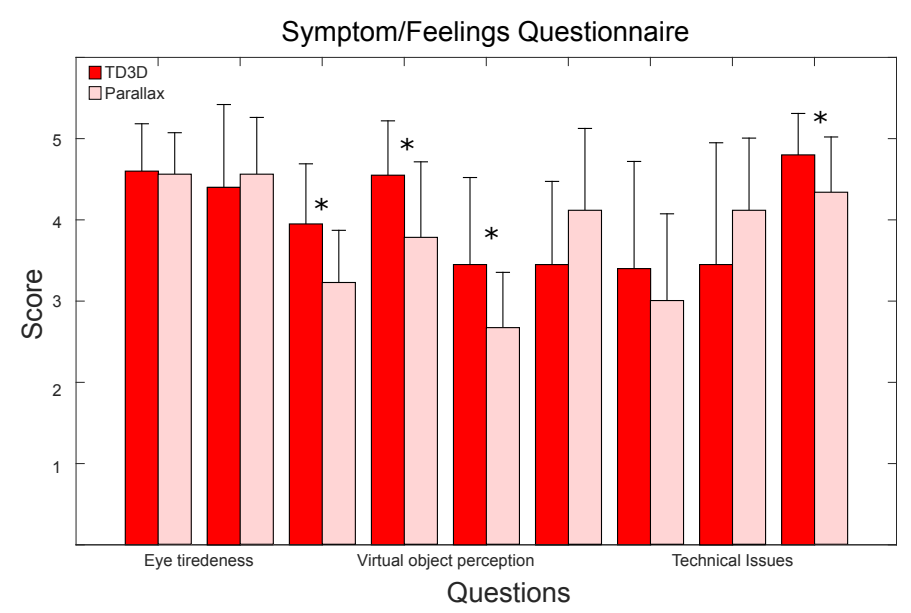

Fig. 6. Results from symptom/feelings questionnaire. Reported scores averaged across subjects are plotted for each of the nine questions. The dark red bars represent the scores for the stereoscopic TD3D sessions and the light red bars represent the scores for the motion parallax sessions. Error bars represent standard deviations across observers; * denotes a significance level of $p<0.025$ for within-subjects analysis.

\section{CONCLUSION}

In this paper, we have proposed a "virtual holographic" system, which is able to correctly render virtual objects to an observer moving around them, as if it were a real glass showcase. We have considered and compared two visualization techniques: one based on stereoscopic rendering and one based on motion parallax. The stereoscopic rendering technique takes into account the tracked position of the eyes of the observers to continuously update the stereoscopic views, and to allow a correct perception of the virtual contents. We have used off-the-shelf devices to realize the prototype, in particular a Microsoft Kinect to detect and track the eyes' positions, and three stereoscopic monitors to show the virtual contents. The scalability of the developed system allows us to increase the number of monitors, in order to obtain larger installations.

The developed system has been validated through an experimental session, conducted by 20 subjects, who tested the prototype with both the visualization techniques independently, by evaluating the visual fatigue, the realism and the usability of the virtual contents. The results show that the two visualization techniques produce both small eye tiredness in the observer, and a similar pleasure in using the system. The analysis of the scores given by the users shows how the perception of virtual objects is better for the stereoscopic visualization technique.

Though the system could be further improved (e.g. some issues related to its stability and latencies have to be solved), we can conclude that stereoscopic cues improve the user's experience and can be used to create a "virtual hologram" effect.

\section{REFERENCES}

[1] E. European Commission, "Mapping of cultural heritage actions in european union policies, programmes and activities," European Commission, Tech. Rep., 2014.

[2] A. Gruen, F. Remondino, and L. Zhang, "Image-based automated reconstruction of the Great Buddha of Bamiyan, Afghanistan," IEEE Computer Society Conference on Computer Vision and Pattern Recognition Workshops, vol. 1, 2003.

[3] M. Levoy, K. Pulli, B. Curless, S. Rusinkiewicz, D. Koller, L. Pereira, M. Ginzton, S. Anderson, J. Davis, J. Ginsberg, J. Shade, and D. Fulk, "The digital Michelangelo project: 3D scanning of large statues," ser. SIGGRAPH '00, 2000, pp. 131-144.

[4] M. Carrozzino and M. Bergamasco, "Beyond virtual museums: Experiencing immersive virtual reality in real museums," Journal of Cultural Heritage, vol. 11, no. 4, pp. 452 - 458, 2010.

[5] S. Styliani, L. Fotis, K. Kostas, and P. Petros, "Virtual museums, a survey and some issues for consideration," Journal of cultural Heritage, vol. 10, no. 4, pp. 520-528, 2009.

[6] N. Magnenat-thalmann and G. Papagiannakis, "Virtual worlds and augmented reality in cultural heritage applications," in International Workshop on Recording, Modeling and Visualization of Cultural Heritage, 2005.

[7] N. Di Blas and C. Poggi, "3D for cultural heritage and education: evaluating the impact," in Museums and the Web 2006 Conference, 2006, pp. 22-25.

[8] R. A. Earnshaw, Virtual reality systems. Academic press, 2014.

[9] T. Agocs, T. Balogh, T. Forgacs, F. Bettio, E. Gobbetti, G. Zanetti, and E. Bouvier, "A large scale interactive holographic display," in Virtual Reality Conference, 2006, 2006, pp. 311-311.

[10] H. Horimai, D. Horimai, T. Kouketsu, P. Lim, and M. Inoue, "Full-color 3D display system with 360 degree horizontal viewing angle," 2010, pp. 7-10.

[11] R. T. Held and M. S. Banks, "Misperceptions in stereoscopic displays: a vision science perspective," in Proceedings of the 5th symposium on Applied perception in graphics and visualization, ser. APGV '08, 2008, pp. 23-32.

[12] M. Chessa, M. Garibotti, A. Canessa, A. Gibaldi, S. P. Sabatini, and F. Solari, "A stereoscopic augmented reality system for the veridical perception of the 3D scene layout," in International Conference on Computer Vision Theory and Applications (VISAPP), 2012, pp. 15-23.

[13] F. Solari, M. Chessa, M. Garibotti, and S. P. Sabatini, "Natural perception in dynamic stereoscopic augmented reality environments," Displays, vol. 34, no. 2, pp. 142-152, 2013.

[14] J. Xue, G. Zhao, and D. Tan, "A motion parallax rendering approach to real-time stereoscopic visualization for aircraft virtual assembly," in Virtual Reality and Visualization (ICVRV), 2012 International Conference on, Sept 2012, pp. 1-6.

[15] B. Rogers and M. Graham, "Similarities between motion parallax and stereopsis in human depth perception," Vision Research, vol. 22, no. 2, pp. $261-270,1982$.

[16] S. Mizuno, M. Tsukada, and Y. Uehara, "A stereoscopic CG system with motion parallax and its digital contents for science museums," in SignalImage Technology Internet-Based Systems (SITIS), 2013 International Conference on, 2013, pp. 378-384.

[17] A. Canessa, M. Chessa, A. Gibaldi, S. P. Sabatini, and F. Solari, "Calibrated depth and color cameras for accurate 3D interaction in a stereoscopic augmented reality environment," Journal of Visual Communication and Image Representation, vol. 25, no. 1, pp. 227 - 237, 2014.

[18] R. M. Taylor, II, T. C. Hudson, A. Seeger, H. Weber, J. Juliano, and A. T. Helser, "VRPN: A device-independent, network-transparent VR peripheral system," in Proceedings of the ACM Symposium on Virtual Reality Software and Technology, 2001, pp. 55-61. 